\title{
Antiinflammatory effects of bromodomain and extraterminal domain inhibition in cystic fibrosis lung inflammation
}

\author{
Kong Chen, ${ }^{1}$ Brian T. Campfield, ${ }^{1,2}$ Sally E. Wenzel, ${ }^{3}$ Jeremy P. McAleer, ${ }^{1}$ James L. Kreindler, ${ }^{4}$ \\ Geoffrey Kurland, ${ }^{5}$ Radha Gopal, ${ }^{1}$ Ting Wang, ${ }^{6}$ Wei Chen, ${ }^{6}$ Taylor Eddens, ${ }^{1}$ Kathleen M. Quinn, ${ }^{1}$ \\ Mike M. Myerburg, ${ }^{7}$ William T. Horne, ${ }^{1}$ Jose M. Lora, ${ }^{8}$ Brian K. Albrecht, ${ }^{8}$ Joseph M. Pilewski, ${ }^{7}$ \\ and Jay K. Kolls' \\ 'Richard King Mellon Foundation Institute for Pediatric Research, Children's Hospital of Pittsburgh of University of \\ Pittsburgh Medical Center (UPMC), ${ }^{2}$ University of Pittsburgh, Division of Pediatric Infectious Diseases, Department of \\ Pediatrics, ${ }^{3}$ University of Pittsburgh Asthma Institute at UPMC, University of Pittsburgh School of Medicine, Pittsburgh, \\ Pennsylvania, USA. ${ }^{4}$ Department of Pediatrics, Children's Hospital of Philadelphia, Philadelphia, Pennsylvania, USA. \\ ${ }^{5}$ Division of Pediatric Pulmonology, Children's Hospital of Pittsburgh of UPMC, 'Division of Pulmonary Medicine, Children's \\ Hospital of Pittsburgh of UPMC, University of Pittsburgh School of Medicine, Pittsburgh, Pennsylvania, USA. \\ 'Division of Pulmonary, Allergy, and Critical Care Medicine, UPMC, Pittsburgh, Pennsylvania, USA. \\ ${ }^{8}$ Constellation Pharmaceuticals Inc., Cambridge, Massachusetts, USA.
}

Significant morbidity in cystic fibrosis (CF) results from chronic lung inflammation, most commonly due to Pseudomonas aeruginosa infection. Recent data suggest that IL-17 contributes to pathological inflammation in the setting of abnormal mucosal immunity, and type 17 immunity-driven inflammatory responses may represent a target to block aberrant inflammation in CF. Indeed, transcriptomic analysis of the airway epithelium from CF patients undergoing clinical bronchoscopy revealed upregulation of IL-17 downstream signature genes, implicating a substantial contribution of IL-17-mediated immunity in CF lungs. Bromodomain and extraterminal domain (BET) chromatin modulators can regulate T cell responses, specifically Th17-mediated inflammation, by mechanisms that include bromodomain-dependent inhibition of acetylated histones at the IL17 locus. Here, we show that, in vitro, BET inhibition potently suppressed Th17 cell responses in explanted CF tissue and inhibited IL-17-driven chemokine production in human bronchial epithelial cells. In an acute $P$. aeruginosa lung infection murine model, BET inhibition decreased inflammation, without exacerbating infection, suggesting that BET inhibition may be a potential therapeutic target in patients with CF.

Conflict of interest: J.M. Lora was an employee of Constellation Pharmaceuticals Inc.

Submitted: February 18, 2016 Accepted: May 31, 2016 Published: July 21, 2016

Reference information: JCI Insight. 2016;1(11):e87168. doi:10.1172/jci.insight.87168.

\section{Introduction}

Patients with cystic fibrosis (CF) suffer chronic respiratory infection, most commonly due to Pseudomonas aeruginosa (1). The ion transport defect leads to chronic infection in the lung and exuberant airway inflammation that result in lung parenchymal and airway damage associated with increased morbidity and mortality. Consistent with this, enhanced constitutive activation of NF- $\mathrm{KB}$ in $\mathrm{CF}$ epithelial cells has been observed, which is partially responsible for the recruitment of neutrophils to the pulmonary mucosa (2). Recently, the cytokine IL-17 (a regulator of NF- $\mathrm{KB}$ in bronchial epithelial cells) has been associated with chronic lung disease, including $\mathrm{CF}$ and chronic obstructive pulmonary disease (COPD) $(3,4)$. We have previously reported increased levels of IL-17 in the sputum during CF exacerbations (5) and identified that CD4+ $\mathrm{T}$ cells, particularly Th17 cells, are a critical source of IL-17 in the CF lung (6). Indeed, $P$. aeruginosa antigen stimulation of mediastinal lymph nodes from patients with CF induces proliferation of Th17 memory cells, suggesting that $P$. aeruginosa can initiate adaptive T cell responses (6). While IL-17-mediated inflammation is critical for host defense against extracellular pathogens, such as $K$. pneumoniae and $C$. albicans (1), our recent data suggested a detrimental role of the IL-17 pathway in a chronic $P$. aeruginosa lung infection model (7). Additionally, we demonstrated that IL-17 regulates $\mathrm{HCO}^{-}$transport in normal human bronchial epithelial cells in a cystic fibrosis transmembrane 
A
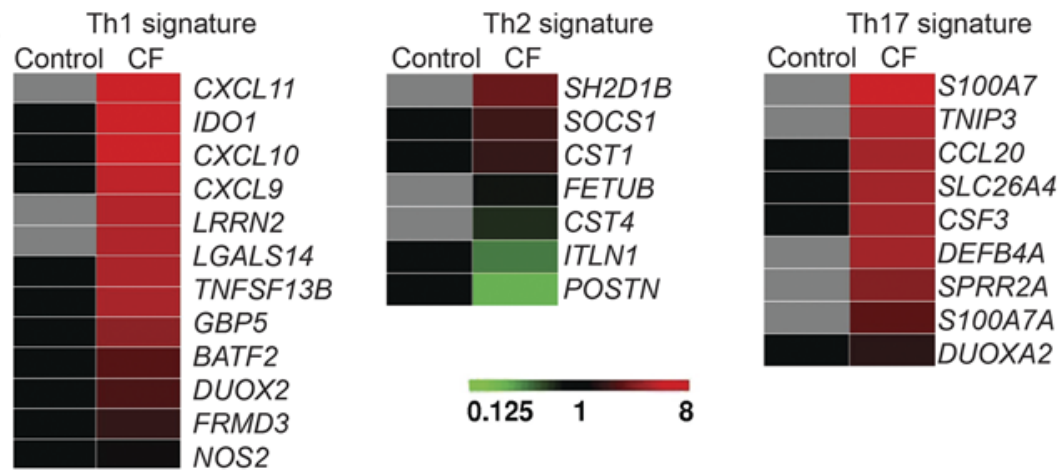

B
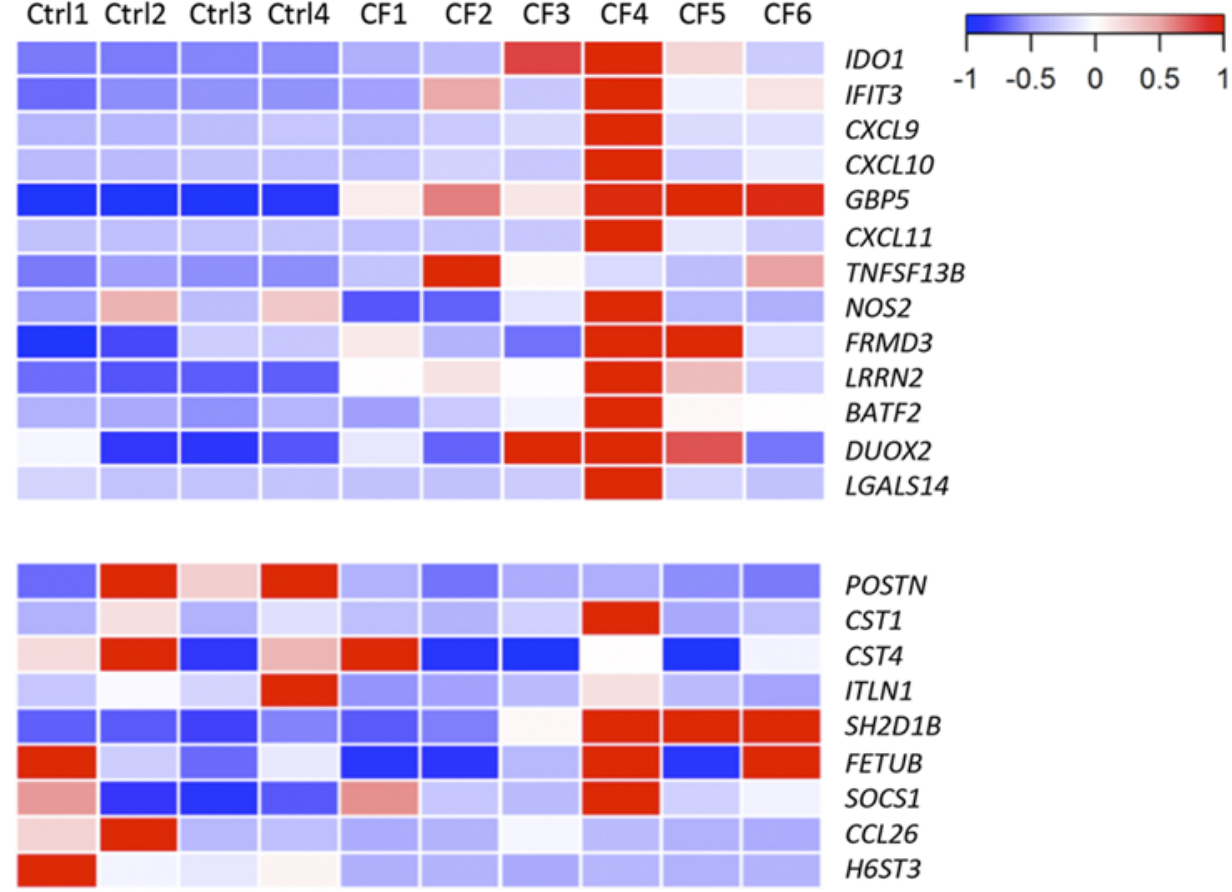

POSTN

CST1

CST4

ITLN1

$S H 2 D 1 B$

FETUB

SOCS1

CCL26

H6ST3

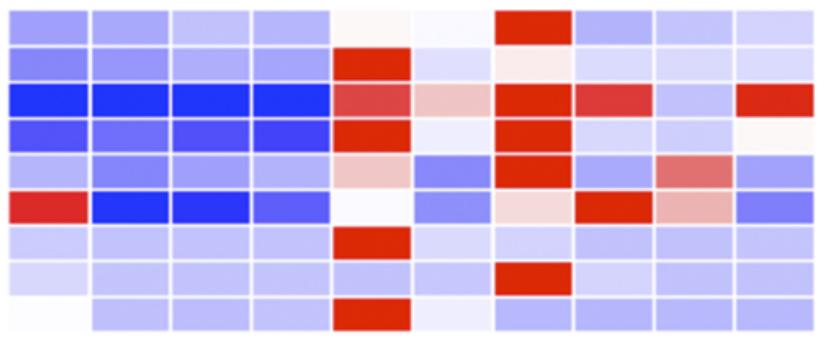

SLC26A4

CCL2O

TNIP3

CSF3

SPRR2A

DUOXA2

S100A7

DEFB4

S100A7A
Figure 1. Enriched T cell signatures are present in CF epithelium. (A) RNA-seq heat maps of CF bronchial brushings vs. control bronchial brushings. Th1, Th2, and Th17 gene signatures determined by RNA-seq from RNA obtained from cytokine-stimulated NHBE cells are shown. (B) T cell signatures in bronchial brushings from individual control and CF patients. Donor demographics are summarized in Supplemental Tables 1 and 2.

conductance regulator-dependent (CFTR-dependent) fashion (8), and others have shown that $\mathrm{HCO}^{-}$is essential for antimicrobial activity in the CF airway (9). Thus, in CF, IL-17 likely contributes to pathological inflammation in the setting of abnormal mucosal immunity (1), and Th17/IL-17-driven inflammatory responses may represent a target to attenuate chronic lung inflammation in CF. Bromodomain and extraterminal (BET) domain chromatin modulators are able to regulate $\mathrm{T}$ cell responses and have been shown to critically contribute to Th17 function by mechanisms that include bromodomain-dependent interpretation of acetylated histones at key genomic sites, such as the IL17 locus (10). Indeed, pharmacological inhibition of BET domains has been shown to protect mice from experimental autoimmune encephalomyelitis, a preclinical model of multiple sclerosis, by suppressing Th17/IL-17driven inflammation (10). Thus, we hypothesized that pharmacologic BET inhibition may provide a novel and effective therapy for treatment of inflammation in $\mathrm{CF}$ lung disease.

\section{Results}

T cell signatures in CF lung epithelium. To study lung epithelial inflammatory responses in CF patients in an unbiased way, we utilized transcriptomic analyses of human bronchial epithelial (HBE) cells treated with various cytokines. In prior RNA sequencing (RNA-seq) experiments (4), we found that HBE cells express all of the receptors necessary to respond to effector cytokines made by $\mathrm{T}$ cells in the submucosa, such as IFN- $\gamma$ (Th1), IL-13 (Th2), or IL-17 (Th17). Based on this, we treated normal HBE (NHBE) cells with these cytokines to identify cytokine-specific signatures (Supplemental Figure 1; supplemental material available online with this article; doi:10.1172/jci.insight.87168DS1) in the epithelium. CXCL9, CXCL10, and $C X C L 11$ were highly induced by IFN- $\gamma$ treatment, whereas DEFB4, CSF3, and CCL20 were found in 

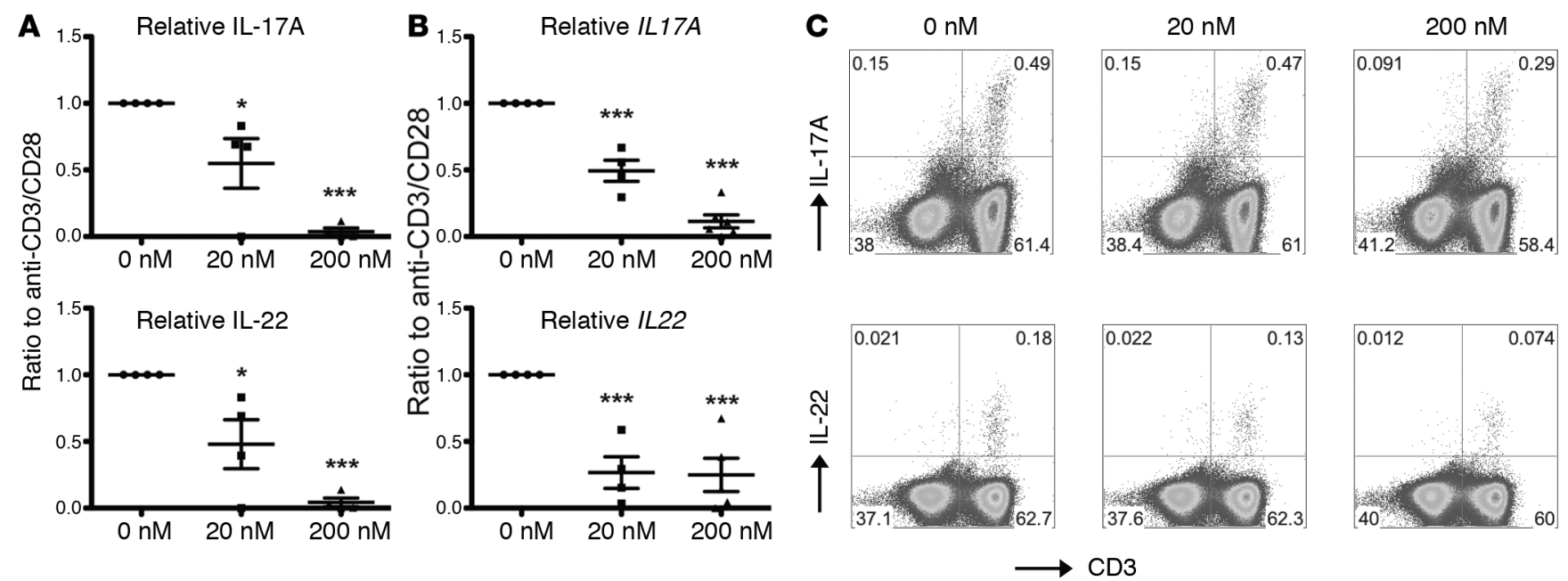

Figure 2. CPI-203 potently suppresses Th17 cytokine production by T cells from CF lungs. (A) Mononuclear cells from CF lung explants were stimulated with human T cell expander beads (anti-CD3/anti-CD28) for 4 days in the presence of indicated concentrations of CPI-203. Tissue culture supernatants were harvested and IL-17A and IL-22 were measured by ELISA. Data are represented by normalization against conditions without the compound for each donor. Donor genotypes are summarized in Supplemental Table 3. (B) After the supernatants were harvested, cells were harvested in TriZol for RNA extraction, and gene expression was determined by real-time RT-PCR. Data are represented by normalization against conditions without the compound for each donor. (C) Mononuclear cells from CF lung explants were restimulated with PMA/ionomycin in the presence of Golgi-plug for 4 hours before harvesting for intracellular cytokine staining analysis. Representative FACS plots of mLN cells are shown. ${ }^{*} P<0.05,{ }^{* * *} P<0.0005$ by ANOVA.

the IL-17 signature, consistent with prior findings $(11,12)$. IL-13 treatment significantly enhances type 2 inflammation-related genes, including CCL26, ITLN1, and POSTN (13-15). We then conducted RNAseq in bronchial brush samples from patients with $\mathrm{CF}$ collected during clinical exacerbation and used cytokine gene signatures obtained in HBE cells to mine the types of inflammation in the CF bronchial brush samples. We observed robust evidence of both Th1 and Th17 signatures in CF patients compared with non-CF controls as a group (Figure 1A). Th1 signature genes, such as IDO1, GBP5, TNFSF13B, and $L R R N 2$, as well as Th17 signature genes, including TNIP3, CSF3, and CCL20, were significantly upregulated in CF patients, while one Th2 signature gene (HS6ST3) was significantly decreased in CF (Supplemental Figure 2). It is worth noting that POSTN, a biomarker used in anti-IL-13 monoclonal antibody clinical trials in asthma (16), was not upregulated in CF brushes, suggesting that type 2 inflammation is not the predominant pathological response in these CF airways. When each patient's signature was compared individually, stronger Th1 and Th17 but not Th2 signatures were also found in CF patients compared with non-CF controls (Figure 1B). These observations are consistent with our previous findings $(5,6)$ that proinflammatory Th17 cells and type 17 cytokines are present in the CF lungs and these inflammatory responses are altering gene expression in lung epithelium in vivo.

BET inhibition in T cellsfrom CF lungs. BET proteins have been shown to critically contribute to Th17 lineage differentiation from human and murine $\mathrm{CD}_{4}^{+}$naive $\mathrm{T}$ cells and, importantly, to control cytokine production in differentiated Th17 cells (10). We thus sought to investigate the effect of small-molecule BET inhibitors in suppressing proinflammatory cytokine production from $\mathrm{T}$ cells recovered from the lungs of patients with $\mathrm{CF}$ lung disease undergoing lung transplantation. We isolated lung parenchymal mononuclear cells from explanted CF lung tissue and stimulated these cells in the presence or absence of a BET inhibitor (CPI-203) (17-19). Signature Th17 effector cytokines released in the tissue culture supernatant after anti-CD3/anti-CD28 stimulation, such as IL-17A and IL-22, were significantly inhibited by CPI-203 in a dose-dependent manner (Figure 2A). This suppression appeared to occur at the level of transcription, as indicated by real-time RT-PCR analysis (Figure 2B). Similarly, FACS analyses demonstrated that $\mathrm{CD} 3^{+} \mathrm{T}$ cells were the major source of IL-17A and IL-22, both in the lymph nodes (Figure 2C) and the lung tissues (data not shown). Interestingly, and consistent with a previous report (19), our data suggested that BET bromodomain inhibition also resulted in the modulation of the Th1 lineage, as the signature cytokine IFN- $\gamma$ was also suppressed. Taken together, these data suggested that $\mathrm{T}$ cell cytokines associated with inflammation in the $\mathrm{CF}$ lung can be suppressed by BET inhibition.

BET inhibition in CF epithelium. Effector cytokines IL-17A, IL-17F, and IL-22 produced by Th17 cells stimulate pulmonary epithelial cells to produce a number of chemokines (e.g., IL-8) and cytokines 

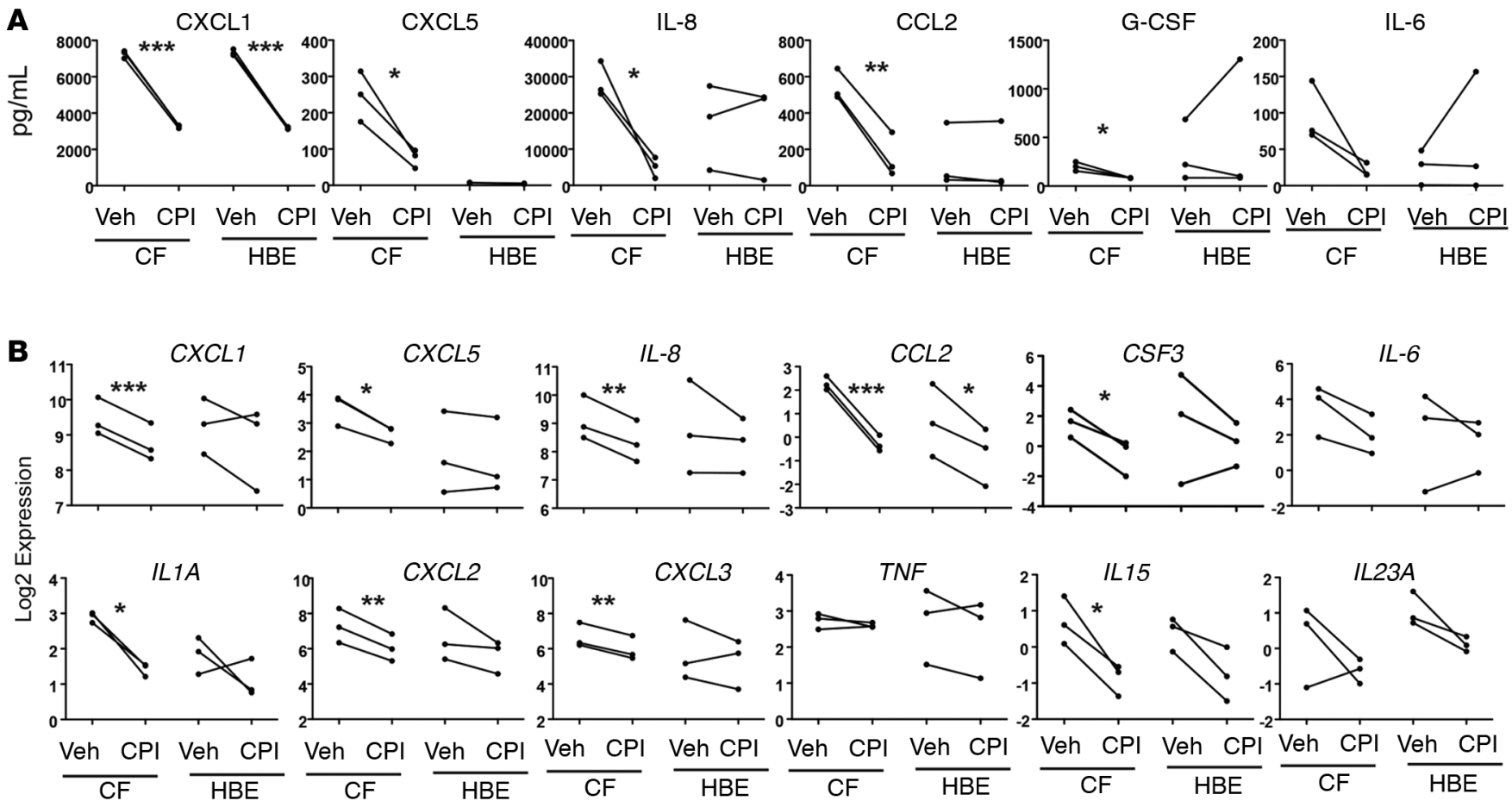

Figure 3. CPI-203 potently suppresses Th17 downstream chemokines and cytokines in HBE cells. HBE cell lines from 3 CF and 3 non-CF (labeled as HBE) donors were established in the air-liquid interphase culture and treated with vehicle (Veh) or $200 \mathrm{nM} \mathrm{CPI-203} \mathrm{(CPI)} \mathrm{for} 3$ days. (A) Culture supernatants were harvested for analysis on proinflammatory chemokines and cytokines by Luminex, and (B) RNA-seq was conducted on the cells and relative gene expression is shown. ${ }^{*} P<0.05,{ }^{* *} P<0.005,{ }^{* *} P<0.0005$ by ANOVA.

(e.g., G-CSF) that attract, activate, and prolong the life span of neutrophils. The prolonged exposure of Th17 cytokines could potentially lead to epigenetic modifications in epithelial cells resulting in constitutive transcriptional activation of $I L 8$ and CSF3. To investigate whether BET proteins could be involved in the interpretation of changes in chromatin consistent with that hypothesis, we performed RNA-seq on $\mathrm{CF}$ and non-CF HBE cells. BET proteins BRD2 and BRD4 were highly expressed in these cells, and their transcript levels were unchanged upon treatment with CPI-203 (Supplemental Figure 3A). To test if BET inhibition could suppress epithelial chemokine and cytokine production, we exposed these cells to vehicle and CPI-203. CXCL1, CXCL5, IL-8, and CCL2 production was consistently inhibited by CPI-203 among different donors as well as G-CSF and IL-6 (Figure 3A). RNA-seq analysis on the same cells revealed that the suppression of these chemokines and cytokines occurred at the transcript level as well (Figure 3B), while other proinflammatory cytokines, such as $T N F$, were not sensitive to BET inhibition, since it is known to be posttranslationally modified (10). Heat map and biclustering of 1,808 differentially expressed genes showed a dramatic effect on CF cells by CPI-203 (Supplemental Figure 3B). Proinflammatory genes, such as CXCL1, CXCL2, CXCL5, IL1A, IL15, and CCL2, were markedly upregulated in CF at baseline, as indicated in Supplemental Figure 3B. CPI-203 treatment inhibited gene expression in the non-CF cells similarly though to a lesser degree (Supplemental Figure 3C), suggesting that $\mathrm{CF}$ epithelial cells are more sensitive to BET inhibition. We speculate this may be due to the epigenetic changes in $\mathrm{CF}$ lungs, which may alter chromatin accessibility in $\mathrm{CF}$ cells, given that both $\mathrm{CF}$ and non-CF cells express comparable levels of BRD2 and BRD4 (Supplemental Figure 3A). KEGG pathway analysis also revealed that additional pathways were influenced by CPI-203 in CF cells compared with non-CF cells (Supplemental Figure 3D), and Ingenuity Pathway Analysis highlighted the involvement of several immune-related pathways, including LPS/IL-1-mediated inhibition of RXR function, altered $\mathrm{T}$ cell and $\mathrm{B}$ cell signaling in rheumatoid arthritis, and Toll-like receptor signaling (Supplemental Figure 3E and Supplemental Table 4). These data suggest that BET inhibitors not only regulate $\mathrm{T}$ cell responses in $\mathrm{CF}$ lungs, but also epithelial chemokine expression, suggesting a fundamental role of BET proteins in the orchestration of pathogenic inflammation in the lungs of $\mathrm{CF}$ patients. 

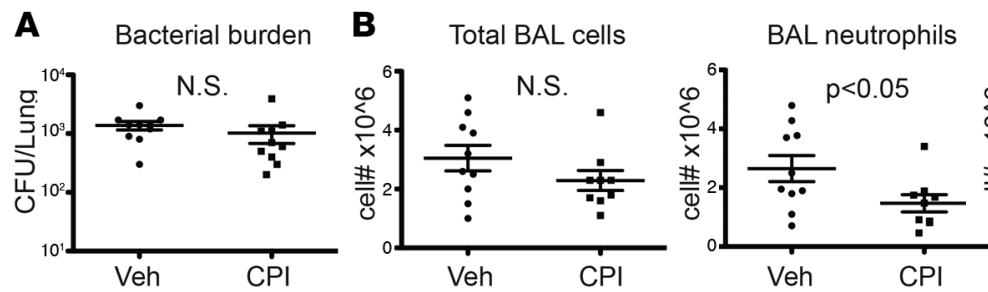

BAL macrophages
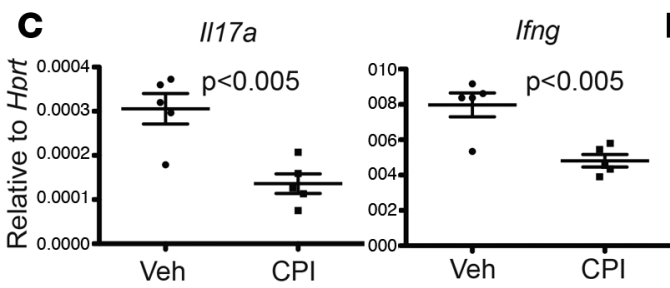

D IL-17A+ cells/lobe
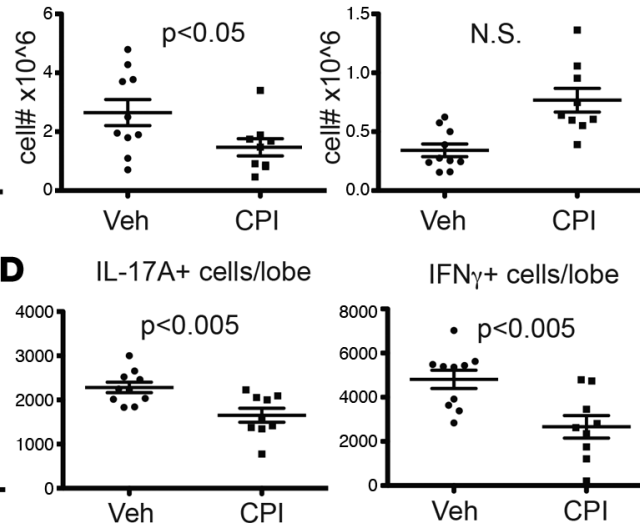

IFN $\gamma+$ cells/lobe
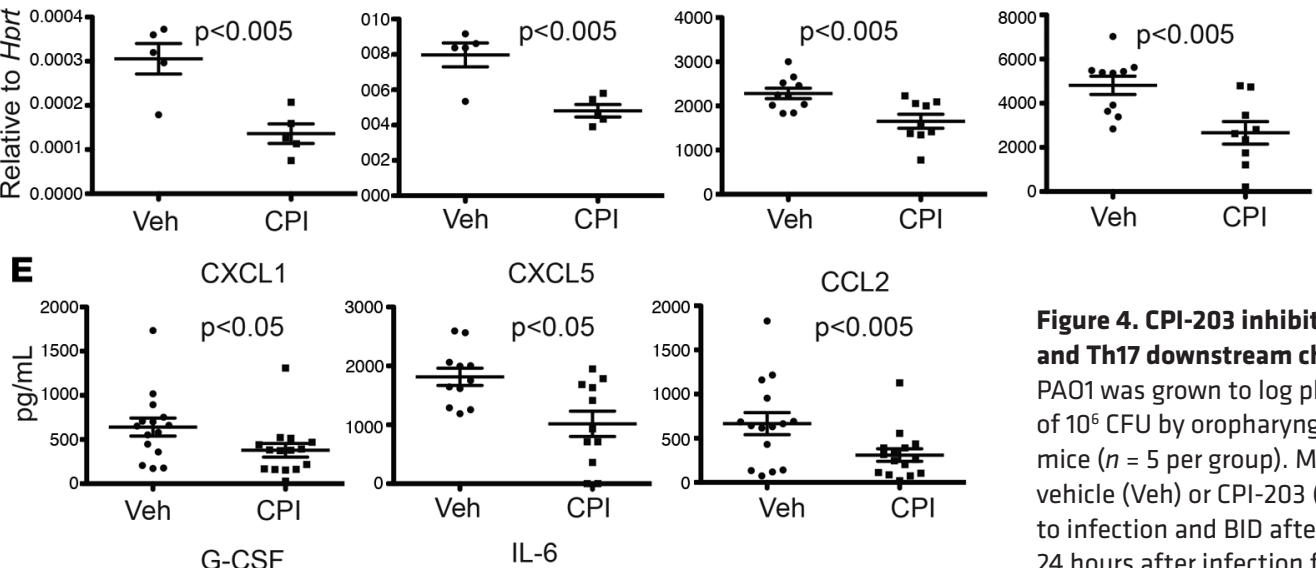

Figure 4. CPI-203 inhibits the production of Th17 cytokines and Th17 downstream chemokines and cytokines in vivo. PA01 was grown to log phase and then inoculated at a dose of $10^{6} \mathrm{CFU}$ by oropharyngeal inoculation into male $\mathrm{C57 \textrm {BI } / 6}$ mice ( $n=5$ per group). Mice were randomized to receive vehicle (Veh) or CPI-203 (CPI) $(2.5 \mathrm{mg} / \mathrm{kg}) 60$ minutes prior to infection and BID afterwards. (A) Lung bacterial burdens 24 hours after infection from control vehicle- and CPI-treated mice were determined by CFU and (B) BAL cell numbers were determined by quick-diff stain. (C) Gene expression in the lung was determined by RT-PCR and (D) total IL-17- and IFN- $\gamma$ producing cells were determined by intracellular staining. (E) Proinflammatory chemokines and cytokines in the whole-lung homogenates were determined by Luminex. $P$ values were calculated by student $t$ test.

BET inhibition on P. aeruginosa induced inflammation in vivo. Given that IL-17 plays a critical role in host defense against many extracellular pathogens, it is crucial to examine whether inhibiting IL-17 responses by BET inhibition would affect the pathogen colonization in CF. Importantly, a potential concern is that reduced airway inflammation by BET inhibition might result in exacerbated infection in the lungs of $\mathrm{CF}$ patients. To explore this in vivo, we dosed CPI-203 in a $P$. aeruginosa acute lung infection model. Mice were randomized to receive vehicle or CPI-203 $(2.5 \mathrm{mg} / \mathrm{kg}) 60$ minutes prior to infection with $P$. aeruginosa (PAO1) and twice daily thereafter. Notably, CPI-203 treatment did not exacerbate bacterial growth (Figure $4 \mathrm{~A}$ ) but resulted in a significant reduction in the recruitment of inflammatory cells, in particular neutrophils (Figure 4B), in the bronchial alveolar lavage fluid, which was associated with the decreased expression of Il17a and Ifng (Figure 4, C and D). Consistent with what we observed in the in vitro NHBE RNA-seq data, levels of chemokine proteins, such as CXCL1, CXCL5, and CCL2, were also decreased in the whole lung homogenates by CPI-203 treatment (Figure 4E). Further, IL-17-driven cytokines, such as G-CSF and IL-6, also showed a trend toward decrease in the lungs from CPI-203-treated mice (Supplemental Figure 4). Furthermore, CPI-203 did not affect the long-term survival of PAO1-infected mice, while neutrophil depletion prior to the infection resulted in substantial mortality (Supplemental Figure 5). There was also a mild attenuation of PAO1-induced inflammation by CPI-203 treatment compared with the vehicle (Supplemental Figure 6). These data are inconsistent with our previous finding that Th17 responses play more of a pathological role than a protective one in $P$. aeruginosa infection (7). Together, these studies show that during acute $P$. aeruginosa lung infection in vivo, CPI-203 treatment reduced IL-17-driven inflammation, without exacerbating infection.

BET inhibition and fungal infection. In addition to Pseudomonas, patients with CF are often colonized with Aspergillus fumigatus, and Th17 cells have been shown to play a critical role in mediating mucosal immunity against this pathogen (20). To assess whether CPI-203 treatment would affect A. fumigatusinduced inflammation and fungal growth, we employed an $A$. fumigatus lung infection model. Similar to the $P$. aeruginosa model, mice were randomized to receive vehicle or CPI-203 $(2.5 \mathrm{mg} / \mathrm{kg}) 60 \mathrm{~min}$ - 
A

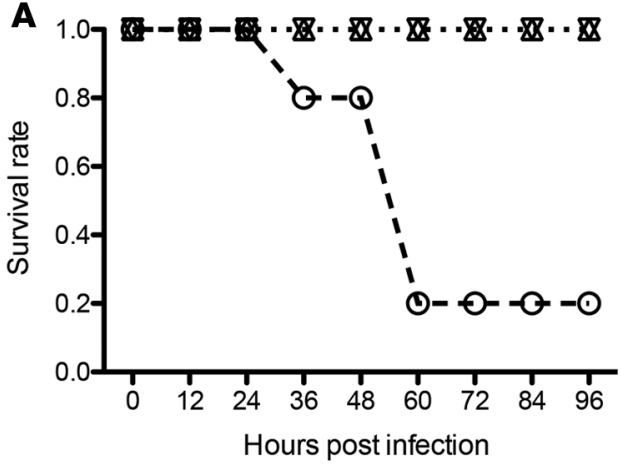

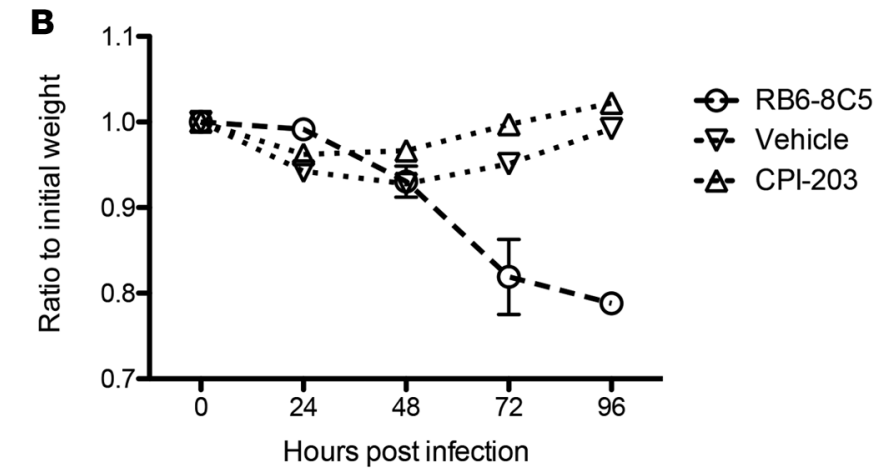
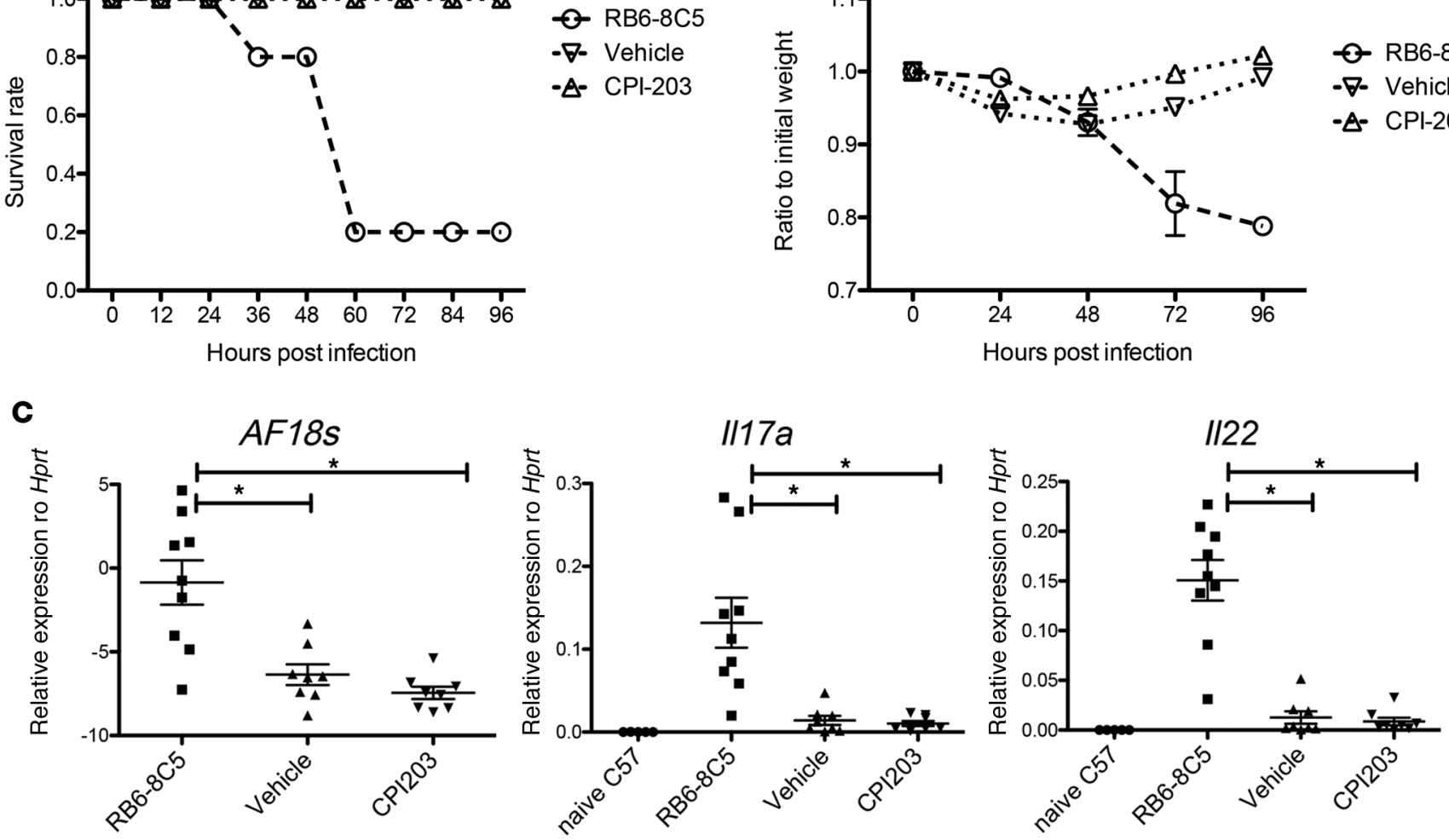

Figure 5. CPI-203 does not exacerbate fungal infection. Female 6- to 8-week-old mice ( $n=5$ per group) were randomized to treatment with vehicle or compound by i.p. administration followed by inoculation with $A$. fumigatus conidia. Mice were infected with $A$. fumigatus via o.p. aspiration (10 ${ }^{7}$ organisms). As a positive control for susceptibility, mice underwent neutrophil depletion by i.p. injection of antibody clone RB6-8C5 (0.6 mg per mouse) 1 day prior to infection. (A) Survival and (B) weight loss of the animals were monitored for 4 days. (C) Fungal burden measured by primers and probes specifically detecting 18s RNA on day 2 after infection and cytokine gene expression in the lungs were determined by real-time RT-PCR. Data were combined from 2 independent experiments. ${ }^{*} P<0.05$ by ANOVA.

utes prior to infection with $A$. fumigatus and twice daily thereafter. CPI-203 treatment did not affect the 4-day survival of $A$. fumigatus-infected mice, while those depleted of $\mathrm{Ly}_{6} \mathrm{G}^{+}$and $\mathrm{Ly}_{6 \mathrm{C}}{ }^{+}$cells (positive control) with RB5-8C5 antibody succumbed to infection (Figure 5, A and B), consistent with previous reports demonstrating an important role of Ly6 $\mathrm{C}^{+}$cells in controlling A. fumigatus clearance (21). The increased morbidity of RB6-8C5-treated mice was associated with higher fungal burdens, whereas CPI203 treatment showed no increase in A. fumigatus infection exacerbation or inflammation (Figure 5C). Our results from these in vivo infection models suggest that, although BET proteins are important drivers of tissue-damaging proinflammatory inflammation, BET inhibition does not adversely affect pathogen clearance in lung infection in these preclinical models.

\section{Discussion}

Pharmacologic therapy in $\mathrm{CF}$ is an area of rapid development. Kalydeco (ivacaftor) has recently been approved by the FDA for the treatment of CF patients with at least one copy of the G511D mutation. Rowe et al. showed that Kalydeco could significantly improve CFTR function, as measured by an increase in predicted $\mathrm{FEV}_{1}$, an increase in $\mathrm{BMI}$, a decrease in sweat chloride concentration, and an increase of the CFQR respiratory score (22). Recently, Wainwright et al. (23) showed that a combination of ivacaftor and lumacaftor further improves $\mathrm{BMI}$ and $\mathrm{FEV}_{1}$ in $\mathrm{CF}$ patients with homozygous F508del mutations. Despite these rapid advances in drug development for treating CF patients, there remains a need for improved understanding of CF disease pathogenesis as well as treatments for the symptoms that may not be affected by the correction of CFTR. Our data showed that both T cells and epithelial cells in $\mathrm{CF}$ are sensitive to BET inhibition (Figures 2 and 3), suggesting the epigenetic modifications in the $\mathrm{CF}$ lung contribute to the enhanced neutrophilic inflammation. If this epigenetic dysregulation is a key contributor to the $\mathrm{CF}$ lung disease, a BET inhibitor may represent a new class of 
antiinflammatory agents to complement CFTR potentiators/correctors. Furthermore, epigenetic dysregulation of chemokine genes has been observed in $\operatorname{COPD}(24,25)$; thus, BET inhibition should be explored in this context as well.

The acute pneumonia model we selected in this study was chosen to mimic the initial phase of $P$. aeruginosa colonization of CF airways. However, at the chronic stage in CF lungs, $P$. aeruginosa forms biofilm and switches to a mucoid phenotype, and the effect of CPI-203 on chronic PA infection warrants careful evaluation in the future. We chose the current dose of $10^{6} \mathrm{CFU}$ PAO1 per mouse based on the current literature $(26,27)$. A dose of $10^{7}$ to $10^{8} \mathrm{CFU}$ per mouse needs to be used to achieve $\mathrm{LD}_{50}$ and at this dose the model is more representative of toxin-induced acute lung injury rather than an acute bacterial infection (28).

CPI-203 is a well-characterized and widely used BET bromodomain inhibitor derived from JQ1, another well-characterized BET bromodomain inhibitor. Both CPI-203 and JQ1 are well-characterized exquisitely selective inhibitors with no known off target effects at biologically relevant concentrations and even at suprapharmacological concentrations $(19,29,30)$. BET bromodomain inhibitors are currently progressing in clinical trials for the treatment of lymphoma, leukemia, and other cancers, and safety data from these studies will be informative to design future studies in other clinical indications. Our data reveal a critical role of BET proteins in the inflammatory response associated with $\mathrm{CF}$, suggesting that these chromatin regulators are fundamental mediators in the inflammatory network observed in the lungs of CF patients. Importantly, these proinflammatory pathways controlled by BET proteins seem to be distinct from the ones implicated in Pseudomonas and Aspergillus clearance (Figure 3A and Figure 4C), opening up the possibility of treating CF-related inflammation with pharmacologic BET inhibitors. Interestingly, two recent reports demonstrated that airway smooth muscle cells are sensitive to BET regulation $(31,32)$, suggesting that BET inhibition could have effects on a variety of cell types in vivo and a synergistic effect could be seen in BET inhibition therapy. Therefore, we propose that BET inhibitors might represent a viable therapeutic option for the control of CF-associated lung disease.

Patients with CF often suffer from inflammatory lung diseases caused by chronic bacterial colonization. Although the constitutive and exaggerated activation of NF- $\mathrm{BB}$ in CF epithelial cells is believed to be in part responsible for the recruitment of large numbers of neutrophils into the pulmonary mucosa, we have indicated that T cells, particularly Th17 cells, are key players in inducing and maintaining the lung inflammation in CF. While eradication of the bacteria in CF airways has been a hallmark of CF patient care, this can be difficult for reasons such as emerging antimicrobial resistance and the formation of drug-impermeable biofilms. Therefore, controlling the inflammatory response is greatly needed since it is the major cause for lung destruction. Corticosteroids, nonsteroidal antiinflammatory drugs such as ibuprofen, cytokines and anticytokines, modulators of intracellular signaling, antioxidants, and protease inhibitors have all been tested in managing CF inflammation, and some have been shown to be effective (33), suggesting that therapy directed at the inflammatory responses in CF airways could be beneficial in CF.

$\mathrm{BET}$ proteins have a pleiotropic nature and play diverse roles in regulating gene transcription, raising safety concerns for the use of BET inhibitors in patients. Indeed, a recent study showed that a brain-permeable inhibitor, I-BET858, selectively suppressed gene expression in neurons and led to the development of an autism-like syndrome in young mice (34). Although no adverse effects of CPI-203 were observed in our study, precautions in choosing highly specific inhibitors to take forward into the clinic should be taken. BET inhibition could also increase the risk of opportunistic infections since our in vivo data showed that CPI-203 suppressed IFN- $\gamma$ (Figure 4, C and D), a cytokine that plays an essential role in tuberculosis and influenza infections (1). Despite these theoretical concerns, our data show promising preclinical evidence supporting further research investigating BET inhibitors to treat chronic lung inflammatory disease.

\section{Methods}

Animal Studies. Eight- to ten-week-old female C57BL/ 6 mice from The Jackson Laboratory were used for in vivo studies. Mice were randomized to receive vehicle or CPI-203 $(2.5 \mathrm{mg} / \mathrm{kg}) 60$ minutes prior to infection with PAO1 (10 $\mathrm{CFU})$ or A. fumigatus $\left(10^{7} \mathrm{CFU}\right)$ by oropharyngeal aspiration and twice daily thereafter.

Cell culture. Lung parenchyma tissues were minced into small pieces with scissors and digested with $1 \mathrm{mg} / \mathrm{ml} \mathrm{Collagenase} \mathrm{(Sigma-Aldrich)} \mathrm{and} 0.1 \mathrm{mg} / \mathrm{ml}$ DNase I (Sigma-Aldrich) for 1 hour at $37^{\circ} \mathrm{C}$. Cells were then passed through a cell strainer to achieve single-cell suspension. Red blood cells were removed by lysis buffer containing $\mathrm{NH}_{4} \mathrm{Cl}$. After 2 washes with cold media, mononuclear cells were 
resuspended in IMDM medium with $10 \%$ FBS. Mediastinal lymph nodes were processed similarly but without Collagenase/DNase I digestion. Cells were then counted by automated cell counter (Countess, Life Technology) and plated in a 24-well plate at a concentration of 1 million/ml in $1 \mathrm{ml}$ medium. Cells were then stimulated in the presence of indicated stimuli in the presence or absence of CPI for 96 hours. Tissue culture supernatants were harvested for ELISA/Luminex and half of the cells were lysed in TriZol for RNA extraction. The rest of the cells were restimulated with PMA/ionomycin for 5 hours in the presence of Golgi-plug, and intracellular IL-17 and IL-22 were determined by FACS.

Fully differentiated HBE cell cultures were derived from lungs removed at the time of lung transplantation of CF patients for CF cells or from the Center for Organ Recovery and Education for non-CF cells. Cells were prepared using previously described methods approved by the University of Pittsburgh IRB (35). Briefly, bronchi from the 2nd to 6th generations were collected, rinsed, and incubated overnight in EMEM at $4^{\circ} \mathrm{C}$. The bronchi were then digested in MEM containing protease XIV and DNase. The epithelial cells were removed and collected by centrifugation and then resuspended in bronchial epithelial growth medium and plated onto collagen-treated tissue culture flasks. When $80 \%-90 \%$ confluence was reached, the passage 0 cells were trypsinized and seeded onto collagen-coated Transwell filters. Upon reaching confluence, the cultures were maintained at an air-liquid interface (ALI) and media was changed basolaterally twice weekly. Cells in ALI culture in quadruplicates were exposed to $200 \mathrm{nM}$ BET inhibitor (CPI-203) basal laterally for 3 days. Basal media were harvested for Luminex analysis and cells were harvested in Qiagen RLT buffer for gene expression analyses.

ELISA. Human IL-17 and IL-22 ELISAs in tissue culture supernatants were performed with kits from R\&D system. The human CXCL5 ELISA kit was purchased from eBioscience.

Luminex. Kits with custom luminex panel, including mouse G-CSF, IL-6, CXCL1 (KC), CXCL5 (LIX), and CCL2, were purchased from EMD Millipore Corporation and the assays were performed per the manufacturer's instructions.

Real-time PCR. RNA was isolated using TriZol reagent (Life Technology), and cDNA was prepared using iScript reverse transcriptase master mix (Bio-Rad). Real-time PCR was carried out with the BioRad CFX96 system using TaqMan PCR Master Mix (Life Technologies) and premixed primers/probe sets (mouse: I117a [Mm00439618_m1], I122 [Mm00444241_m1]. Human: IL17A [Hs00174383_m1], IL22 [Hs01574154_m1]) from Life Technologies.

Flow cytometry. Intracellular staining was performed using BD fix/perm reagents and antibodies were purchased from eBioscience. Data were acquired using FACS LSR II (BD Bioscience) and analyzed by FlowJo software.

RNA-seq analysis. The RNA-seq analysis methodology was published previously (36). The raw RNAseq data have been deposited into the sequencing read archive under SRA accession number SRP075763.

Statistics. All data are presented as the mean \pm SEM. For paired experiments, statistical significance was determined by a 2-tailed, paired $t$ test. A $P$ value of less than 0.05 was considered significant.

Study approval. All animal studies were approved by the Institutional Animal Care and Use Committee of the University of Pittsburgh. Human samples were collected with approval from the University of Pittsburgh IRB.

\section{Author contributions}

$\mathrm{KC}, \mathrm{BTC}$, and JKK designed the experiment and wrote the manuscript. KC, RG, MMM, and JMP performed the experiments using human cells and analyzed the data. KC, BTC, KMQ, and RG generated the mouse data in vivo. SEW, JPM, JLK, GK, TW, WC, TE, and WTH all contributed to the RNA-seq experiment and data analysis. JML and BKA provided the compound and contributed to the manuscript writing.

\section{Acknowledgments}

We thank Stefanie Brown for providing technical assistance for HBE cultures and Wenyu Xiang, Martin Majewski, and Annabel Ferguson for RNA-seq library preparation and sequencing. We also thank Charlie Hatton and Barbara Bryant for comments on the transcriptional analysis. We would like to thank Elizabeth Hartigan for providing the patient demographic information. This work was supported by NIH grants R37HL079142 (to KC and JKK), K08HL128809 (to BTC), and F30AI114146 (to TE) and P01 AI106684 (to SEW) by Cystic Fibrosis Foundation grant KOLLS13I0. KC was also supported by the Research Advisory Committee of Children's Hospital of Pittsburgh of UPMC. 
Address correspondence to: Jay K. Kolls, Department of Pediatrics, Children's Hospital of Pittsburgh of University of Pittsburgh Medical Center, Rangos Research Building, 4401 Penn Avenue, Pittsburgh, Pennsylvania 15224, USA. Phone: 412.692.7710; E-mail: Jay.Kolls@chp.edu.

B.K. Albrecht's present address is: Third Rock Ventures, Cambridge, Massachusetts, USA.

J.M. Lora's present address is: Seres Therapeutics Inc., Cambridge, Massachusetts, USA.

1. Chen K, Kolls JK. T cell-mediated host immune defenses in the lung. Annu Rev Immunol. 2013;31:605-633.

2. Rottner M, Freyssinet JM, Martínez MC. Mechanisms of the noxious inflammatory cycle in cystic fibrosis. Respir Res. 2009;10:23.

3. Alcorn JF, Crowe CR, Kolls JK. TH17 cells in asthma and COPD. Annu Rev Physiol. 2010;72:495-516.

4. Chen K, et al. IL-17RA is required for CCL2 expression, macrophage recruitment, and emphysema in response to cigarette smoke. PLoS One. 2011;6(5):e20333.

5. McAllister F, et al. Role of IL-17A, IL-17F, and the IL-17 receptor in regulating growth-related oncogene-alpha and granulocyte colony-stimulating factor in bronchial epithelium: implications for airway inflammation in cystic fibrosis. J Immunol. 2005;175(1):404-412.

6. Chan YR, et al. Patients with cystic fibrosis have inducible IL-17+IL-22+ memory cells in lung draining lymph nodes. J Allergy Clin Immunol. 2013;131(4):1129.e1

7. Dubin PJ, Martz A, Eisenstatt JR, Fox MD, Logar A, Kolls JK. Interleukin-23-mediated inflammation in Pseudomonas aeruginosa pulmonary infection. Infect Immun. 2012;80(1):398-409.

8. Kreindler JL, et al. Interleukin-17A induces bicarbonate secretion in normal human bronchial epithelial cells. Am J Physiol Lung Cell Mol Physiol. 2009;296(2):L257-L266.

9. Pezzulo AA, et al. Reduced airway surface $\mathrm{pH}$ impairs bacterial killing in the porcine cystic fibrosis lung. Nature. 2012;487(7405):109-113.

10. Mele DA, Salmeron A, Ghosh S, Huang HR, Bryant BM, Lora JM. BET bromodomain inhibition suppresses TH17-mediated pathology. J Exp Med. 2013;210(11):2181-2190.

11. Cooper AM, Khader SA. The role of cytokines in the initiation, expansion, and control of cellular immunity to tuberculosis. Immunol Rev. 2008;226:191-204.

12. Onishi RM, Gaffen SL. Interleukin-17 and its target genes: mechanisms of interleukin-17 function in disease. Immunology. 2010;129(3):311-321

13. Yuan Q, et al. Membrane-bound eotaxin-3 mediates eosinophil transepithelial migration in IL-4-stimulated epithelial cells. Eur J Immunol. 2006;36(10):2700-2714.

14. Kerr SC, et al. Intelectin-1 is a prominent protein constituent of pathologic mucus associated with eosinophilic airway inflammation in asthma. Am J Respir Crit Care Med. 2014;189(8):1005-1007.

15. Jia G, et al. Periostin is a systemic biomarker of eosinophilic airway inflammation in asthmatic patients. J Allergy Clin Immunol. 2012;130(3):647-654.e10.

16. Corren J, et al. Lebrikizumab treatment in adults with asthma. N Engl J Med. 2011;365(12):1088-1098.

17. Sueoka H, Ehara S, Kobayashi H, Arichi T, Komatsu H, inventors; Yoshitomi Pharmaceutical Industries, Ltd., assignee. Thienotriazolodiazepine compounds of the formula (1) \#\#STR1\#\#. US patent 5712274 A. January 27, 1998.

18. Devaiah BN, et al. BRD4 is an atypical kinase that phosphorylates serine2 of the RNA polymerase II carboxy-terminal domain. Proc Natl Acad Sci U S A. 2012;109(18):6927-6932.

19. Moros A, et al. Synergistic antitumor activity of lenalidomide with the BET bromodomain inhibitor CPI203 in bortezomib-resistant mantle cell lymphoma. Leukemia. 2014;28(10):2049-2059.

20. Zelante T, et al. IL-23 and the Th17 pathway promote inflammation and impair antifungal immune resistance. Eur J Immunol. 2007;37(10):2695-2706.

21. Espinosa $\mathrm{V}$, et al. Inflammatory monocytes orchestrate innate antifungal immunity in the lung. PLoS Pathog. 2014;10(2):e1003940.

22. Rowe SM, et al. Clinical mechanism of the cystic fibrosis transmembrane conductance regulator potentiator ivacaftor in G551D-mediated cystic fibrosis. Am J Respir Crit Care Med. 2014;190(2):175-184.

23. Wainwright CE, et al. Lumacaftor-ivacaftor in patients with cystic fibrosis homozygous for Phe508del CFTR. $N$ Engl J Med. 2015;373(3):220-231.

24. Ito $\mathrm{K}$, et al. A molecular mechanism of action of theophylline: Induction of histone deacetylase activity to decrease inflammatory gene expression. Proc Natl Acad Sci U S A. 2002;99(13):8921-8926.

25. Cosio BG, Tsaprouni L, Ito K, Jazrawi E, Adcock IM, Barnes PJ. Theophylline restores histone deacetylase activity and steroid responses in COPD macrophages. J Exp Med. 2004;200(5):689-695.

26. Muir R, et al. Innate Lymphoid Cells Are the Predominant Source of IL-17A during the Early Pathogenesis of Acute Respiratory Distress Syndrome. Am J Respir Crit Care Med. 2016;193(4):407-416.

27. Zou C, et al. Mortality factor 4 like 1 protein mediates epithelial cell death in a mouse model of pneumonia. Sci Transl Med. 2015;7(311):311ra171

28. Balloy V, Verma A, Kuravi S, Si-Tahar M, Chignard M, Ramphal R. The role of flagellin versus motility in acute lung disease caused by Pseudomonas aeruginosa. J Infect Dis. 2007;196(2):289-296.

29. Wong C, et al. The bromodomain and extra-terminal inhibitor CPI203 enhances the antiproliferative effects of rapamycin on human neuroendocrine tumors. Cell Death Dis. 2014;5:e1450. 
30. King B, et al. The ubiquitin ligase FBXW7 modulates leukemia-initiating cell activity by regulating MYC stability. Cell. 2013;153(7):1552-1566

31. Perry MM, Durham AL, Austin PJ, Adcock IM, Chung KF. BET bromodomains regulate transforming growth factor- $\beta$-induced proliferation and cytokine release in asthmatic airway smooth muscle. J Biol Chem. 2015;290(14):9111-9121.

32. Clifford RL, et al. CXCL8 histone $\mathrm{H} 3$ acetylation is dysfunctional in airway smooth muscle in asthma: regulation by BET. Am J Physiol Lung Cell Mol Physiol. 2015;308(9):L962-L972.

33. Chmiel JF, Konstan MW, Elborn JS. Antibiotic and anti-inflammatory therapies for cystic fibrosis. Cold Spring Harb Perspect Med 2013;3(10):a009779.

34. Sullivan JM, et al. Autism-like syndrome is induced by pharmacological suppression of BET proteins in young mice. $J$ Exp Med. 2015;212(11):1771-1781.

35. Myerburg MM, Harvey PR, Heidrich EM, Pilewski JM, Butterworth MB. Acute regulation of the epithelial sodium channel in airway epithelia by proteases and trafficking. Am J Respir Cell Mol Biol. 2010;43(6):712-719.

36. Ray M, et al. RNA-seq in pulmonary medicine: how much is enough?. Am J Respir Crit Care Med. 2015;192(3):389-391. 